\title{
Scedosporium apiospermum endophthalmitis: diffusion-weighted imaging in detecting subchoroidal abscess
}

This article was published in the following Dove Press journal:

Clinical Ophthalmology

20 November 2012

Number of times this article has been viewed

\author{
Sandeep Bhuta \\ Charlie Chia-Tsong Hsu \\ Gigi Nga Chi Kwan \\ Griffith University, School of Medicine, \\ Department of Medical Imaging, Gold \\ Coast Hospital, Southport, Gold \\ Coast, QLD, Australia
}

Purpose: To describe the imaging appearance of Scedosporium apiospermum (S. apiosermum) endophthalmitis in an immunocompetent female who underwent high resolution magnetic resonance imaging (MRI) of the orbits and showed subchoroidal abscess on diffusion-weighted imaging.

Methods: We highlight utility of MRI sequences: diffusion-weighted imaging (DWI), apparent diffusion coefficient (ADC), fluid-attenuated inversion recovery (FLAIR), and contrastenhanced magnetic resonance imaging (CE-MRI) in the detection of a subchoroidal abscess and characterization of the inflammatory change of the uveal tract. Vitreous culture grew S. apiospermum.

Conclusion: Fungal endophthalmitis is a rare but aggressive process. Clinically, it can mimic other disease entities such as neoplasm. To the best of our knowledge, this is the first case that describes the CT and MRI imaging findings of S. apiospermum endophthalmitis. We emphasize the use of DWI and ADC sequences in the detection of subchoroidal abscess.

Keywords: Secosporidium apiospermum, endophthalmitis, subchoroidal abscess, magnetic resonance imaging, diffusion-weighted imaging, contrast-enhanced magnetic resonance imaging

\section{Introduction}

Scedosporium apiospermum (S. apiospermum) is a saprophytic fungus, and is a rare cause of endogenous endophthalmitis affecting mostly immunocompromised patients. ${ }^{1,2}$ A high degree of clinical suspicion is necessary for prompt diagnosis, as orbital infection will often result in loss of vision, and disseminated disease can be fatal. ${ }^{1,3}$ Early imaging helps to assist in establishing the diagnosis and to assess the extent of involvement. Vitreous sampling and laboratory cultures are the only systemic methods for making the diagnosis. To the best of our knowledge, this is the first case in the literature describing imaging features of $S$. apiospermum endophthalmitis with subchoroidal abscess.

\section{Case report}

A 78-year-old female presented with a 5-day history of blurred vision in the right eye, periocular pain, swelling, and photophobia. Past medical history was unremarkable except for recent bronchoscopy and transbronchial biopsy for a suspicious pulmonary nodule. The histopathology and immunohistochemistry confirmed the diagnosis of malignant melanoma.

On ophthalmological examination, visual acuity in her right eye was reduced to hand movements only and in her left eye it was 6/6. Slit lamp examination of her right eye
Correspondence: Sandeep Bhuta Medicine, Griffith University, 108 Nerang Street, Gold Coast Hospital, Gold Coast, Queensland, 4215 Australia

Tel +6I 755198254

Fax +6I 755197964

Email sandeepbhuta@gmail.com 
showed intense inflammatory activity in the anterior chamber with a hazy view of the fundus. The pupil was poorly dilated due to almost $360^{\circ}$ of posterior synechiae. B-scan of the posterior segment revealed vitreous opacities and dense condensations on the posterior vitreous face. Clinical diagnosis of possible endophthalmitis or pseudo tumor was made. As pulmonary nodule biopsy performed a week earlier showed malignant melanoma, choroidal melanoma was included in the differential diagnosis.

Urgent computerized tomography (CT) and magnetic resonance imaging (MRI) examinations were organized. Contrast-enhanced CT showed marked enhancement of the uveal tract on the right with preseptal edema and lateral thickening of the orbital septum (Figure 1), however, there was no retrobulbar fat stranding or posterior chamber abscess detected. Fluid attenuation inversion recovery (FLAIR) axial images demonstrated a definite signal change in the right vitreous chamber and a high signal subchoroidal effusion was seen (Figure 2A). Diffusion-weighted imaging (DWI) and apparent diffusion coefficients (ADC) were the key sequences showing restricted diffusion within the posterior chamber suggestive of subchoroidal abscess or effusion. Effusion was seen as a linear high signal within the right globe laterally extending up to the level of the optic nerve head (Figures $2 \mathrm{~B}$ and $\mathrm{C}$ ). The ADC value from the abscess measured $602 \times 10^{-6} \mathrm{~mm}^{2} / \mathrm{s}$ in comparison to the central portion of $2650 \times 10^{-6} \mathrm{~mm}^{2} / \mathrm{s}$, implying true restriction. On postcontrast $\mathrm{T} 1$ fat saturated images, diffuse enhancement of the thickened sclera and the entire uveal was identified (Figure 3). Also seen was extension of this inflammatory process into the orbital septum as well as the extra-ocular muscles with marked thickening of the right lateral rectus muscle which also enhanced with contrast.

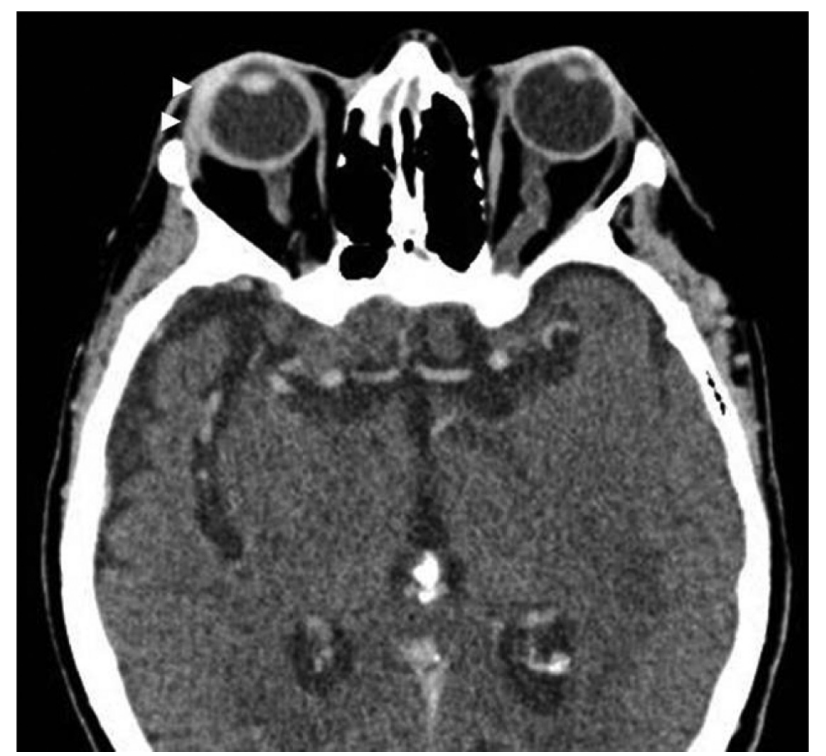

Figure I Contrast-enhanced axial CT of the orbit shows preseptal swelling and thickening of the orbital septum on the right. Note thickening and enhancement of uveal tract on right. No obvious abnormality seen in vitreous chamber.

The extraconal inflammatory change was localized more in the superolateral part of the right orbit. Recent diagnosis of melanoma from a pulmonary nodule caused a diagnostic dilemma and ocular melanoma or melanoma metastases were included in the initial differential diagnoses. MRI immensely helped in narrowing the differential diagnosis as there was no T1 bright lesion seen in the choroid to suggest melanoma.

Cytology from the initial vitreous aspirate did not yield malignant cells and subsequent cultures were negative. Blood tests showed moderate neutrophilia of $8.2 \times 10^{9} / \mathrm{L}$, ESR of $20 \mathrm{~mm} /$ hour, and a normal serum ACE level of 35 U/L. Quantiferon TB Gold, VDRL, and blood cultures were also negative. Toxoplasmosis testing showed $\operatorname{IgG}$
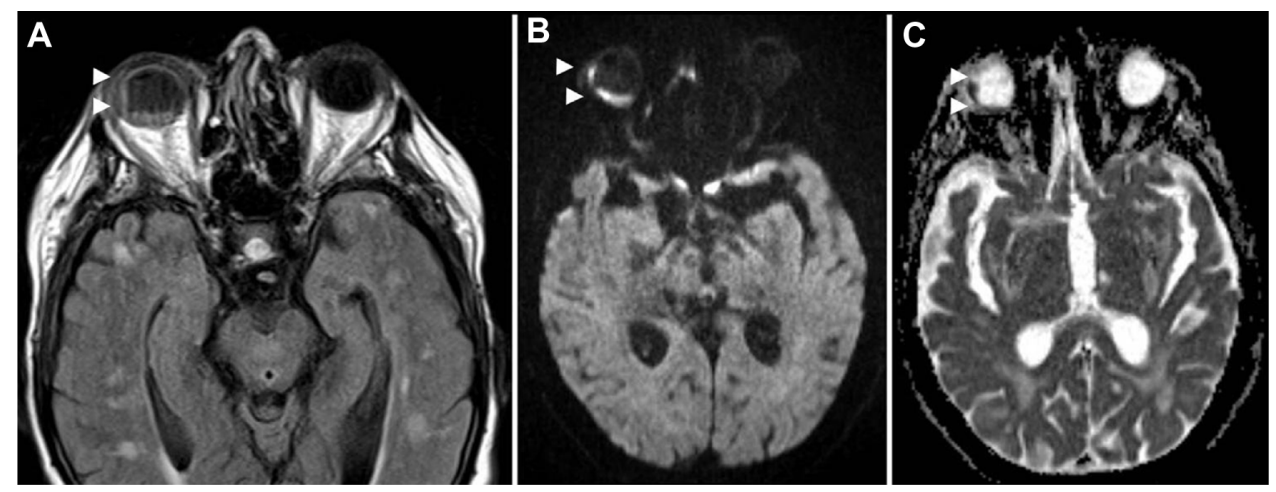

Figure 2 FLAIR axial image (A) depicts high signal in the right vitreous (arrowheads) suggestive of subchoroidal effusion. Note low signal in normal vitreous on the left due to fluid suppression. DWI sequence (B) and corresponding ADC map (C) demonstrate restricted diffusion (bright signal) within the vitreous chamber on the right and confirms a subchoroidal abscess (arrowheads). The mean ADC value in the subchoroidal abscess was $602 \times 10^{-6} \mathrm{~mm}^{2} / \mathrm{s}$, and in the central portion $2650 \times 10^{-6} \mathrm{~mm} / \mathrm{s}$. The left globe was normal, with a mean ADC of $2737 \times 10^{-6} \mathrm{~mm}^{2} / \mathrm{s}$. 


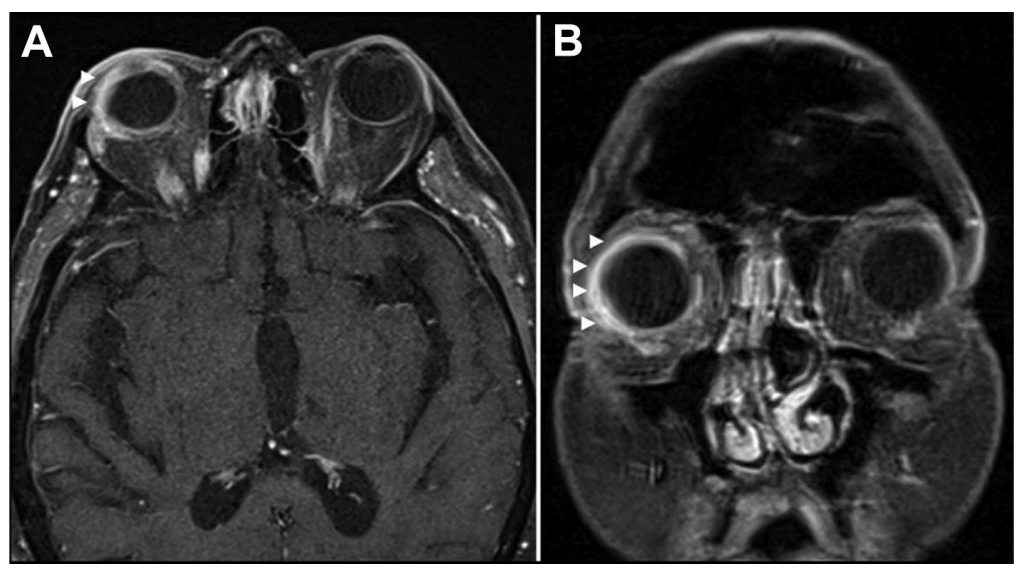

Figure 3 Contrast-enhanced FS axial and coronal TI-weighted images (A, B) reveal enhancement of the uveal tract and the subchoroidal abscess on the right along with preseptal edema. Also note the extension into the retrobulbar fat and extra-ocular muscles, outlining the extent of the inflammatory process.

reactivity with IgM nonreactivity. Culture from a repeated vitreous aspirate sample grew S. apiospermum. Antifungal susceptibility testing showed sensitivity to itraconazole and voriconazole with a minimum inhibitory concentration (MIC) of $0.5 \mu \mathrm{g} / \mathrm{mL}$ and $0.25 \mu \mathrm{g} / \mathrm{mL}$, respectively. The patient was then started on a 6-month regimen of oral voriconazole. Despite therapy, patient's vision showed no improvement and vision remained restricted to hand movements only at the 12-month follow-up.

\section{Discussion}

S. apiospermum is a ubiquitous fungal pathogen in the environment and is most commonly found in soil and polluted water. This organism can infect healthy individuals through direct inoculation of the eye as the result of trauma or a complication of intraocular surgery. In immunocompromised patients, hematogenous spread or local extension of an infective focus is more commonly encountered. Common sites of infectious S. apiospermum in immunocompromised patients include the lungs, joints, central nervous system, sinuses, and ears. ${ }^{2}$ Orbital involvement of this organism has rarely been reported. In our patient, it is difficult to determine whether the endophthalmitis was the result of direct inoculation from the bronchoscopy procedure or hematogenous seeding. It is possible that the patient had an occult respiratory colonization by $S$. apiospermum and the transbronchial biopsy may have precipitated the hematogenous spread. Although our patient was not immunosuppressed, the solitary metastatic melanoma to the lung is a possible complicating factor.

There have been two previous reports of bacterial endophthalmitis and one case of nocardia endophthalmitis featuring DWI restriction confirmed on ADC maps. ${ }^{4-6}$ Our case, together with these two previous case reports, suggests that DWI and ADC sequence is a sensitive method in detection of subchoroidal and subretinal abscesses. In our clinical scenario, it certainly helped to exclude ocular melanoma and was more specific to suggest an inflammatory or infectious condition. The use of contrast-enhanced magnetic resonance imaging (CE-MRI) allowed us to better delineate the extent of the inflammatory process. Most recently, Yu et al demonstrated the usefulness of DWI and CE-MRI in assessing a patient with nocardia endophthalmitis. ${ }^{4}$ In addition, Yu et al were able to demonstrate retinal detachment on $\mathrm{T} 2$-weighted, fat-saturated imaging as a complication to their case. ${ }^{4}$ In our case, T2-weighted sequence was not useful in demonstrating the subchoroidal abscess. Instead, FLAIR sequence was very useful to detect altered contents within the vitreous as a crescent-shaped hyperintensity due to its inherent fluid suppression property. This finding was reinforced on the corresponding DWI and ADC sequences. The usefulness of the FLAIR sequences had been reported in two previous cases of endophthalmitis in the detection of subtle intraocular lesion. ${ }^{5,6}$ Although follow-up imaging was not performed in our case, we speculate that MR imaging has a potential role in monitoring responsiveness to treatment. Rumboldt et al, in a case of Staphylococcus aureus endophthalmitis demonstrated decreased DWI signal intensity with corresponding increase in mean ADC values, reflecting positive treatment response. ${ }^{5}$

Endophthalmitis is often a clinical diagnosis and imaging may not always be mandatory. Multi-sequence MR imaging with T1, DWI/ADC, and FLAIR sequences can assist in confirming clinical diagnosis and excluding neoplastic causes. Diffusion-weighted imaging is sensitive in diagnosing intraocular or subchoroidal abscesses. The FLAIR sequence can detect early inflammatory changes in the vitreous chamber. CE-MRI further characterizes the extent of 
the inflammatory process involving the uveal tract and the extra-ocular muscles. Combined use of DWI and FLAIR may add a degree of confidence and diagnostic specificity in the assessment of patients with clinically suspected infective endophthalmitis.

\section{Disclosure}

The authors report no conflicts of interest in this work.

\section{References}

1. Jain A, Egbert P, McCulley TJ, Blumenkranz MS, Moshfeghi DM. Endogenous Scedosporium Apiospermum endophthalmitis. Arch Ophthalmol. 2007;125(9):1286-1289.

2. Jones J, Katz SE, Lubow M. Scedosporium apiospermum of the orbit. Arch Ophthalmol. 1999;117(2):272-273.
3. McKelvie PA, Wong EY, Chow LP, Hall AJ. Scedosporium endophthalmitis: two fatal disseminated cases of Scedosporium infection presenting with endophthalmitis. Clin Experiment Ophthalmol. 2001;29(5):330-334.

4. Yu E, Laughlin S, Kassel EE, Messner HA, Yucel YH. Nocardial endophthalmitis and subretinal abscess: CT and MR imaging features with pathologic correlation: a case report. AJNR Am J Neuroradiol. 2005;26(5):1220-1222.

5. Rumboldt Z, Moses C, Wieczerzynski U, Saini R. Diffusion-weighted imaging, apparent diffusion coefficients, and fluid-attenuated inversion recovery MR imaging in endophthalmitis. AJNR Am J Neuroradiol. 2005;26(7):1869-1872.

6. Seale M, Lee WK, Daffy J, Tan Y, Trost N. Fulminant endogenous Klebsiella pneumoniae endophthalmitis: imaging findings. Emerg Radiol. 2007;13(4):209-212.
Clinical Ophthalmology

\section{Publish your work in this journal}

Clinical Ophthalmology is an international, peer-reviewed journal covering all subspecialties within ophthalmology. Key topics include: Optometry; Visual science; Pharmacology and drug therapy in eye diseases; Basic Sciences; Primary and Secondary eye care; Patient Safety and Quality of Care Improvements. This journal is indexed on

Submit your manuscript here: http://www.dovepress.com/clinical-ophthalmology-journal

\section{Dovepress}

PubMed Central and CAS, and is the official journal of The Society of Clinical Ophthalmology (SCO). The manuscript management system is completely online and includes a very quick and fair peer-review system, which is all easy to use. Visit http://www.dovepress.com/ testimonials.php to read real quotes from published authors. 